\section{It ain't over until the polymerase falls off}

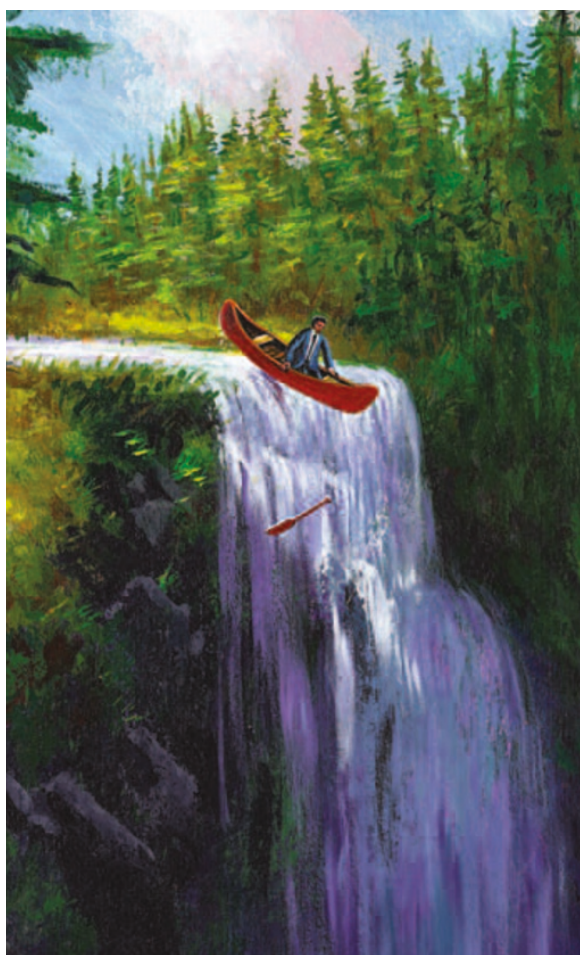

Today, it is widely accepted that RNA chain elongation and termination by RNA polymerase II (pol II) is a complex process that is coordinated with 3 '-end processing and polyadenylation of the primary transcript. Just over 20 years ago, however, the identification of the first elongation factor, TFIIS or SII, provided the initial indication that RNA pol II transcription could be regulated at the level of elongation.

Back in 1973, Natori and colleagues identified SII by its ability to stimulate transcription in vitro and to enable pol II to synthesize long transcripts. Yet its mode of action remained unknown until 1992, when three groups added a considerable piece to the puzzle by providing insights into the mechanism of SII activity. Reines, and Izban and Luse, noticed that the addition of SII caused a shortening of transcripts associated with stalled RNA pol II. They found that, in the presence of SII, the RNA pol II complex can serve as a nuclease, cleaving its nascent transcript from the $3^{\prime}$ end. Wang and Hawley also presented evidence to support these observations, and proposed a possible proofreading role for the activity described. Surprisingly, it was noted that this process leaves the pol II complex intact and the remaining transcript can subsequently be elongated. The nuclease activity that is stimulated by SII helps pol II bypass specific blocks to elongation and therefore increases elongation efficiency.

A question that puzzled the community was how 3 '-end processing was linked to termination. The connection between these processes was established when it became apparent that polyadenylation and transcription termination were dependent on the same DNA sequences at the $3^{\prime}$ ends of genes. A role for poly(A) site cleavage in termination was first established by two groups - Logan and colleagues, and Connelly and Manley. Based on the hypothesis that polyadenylation must be a prerequisite for RNA pol II termination, because this would ensure that mRNAcoding sequences were completely transcribed before a termination event occurred, they introduced several singlebase-pair mutations into the polyadenylation motifs, and showed abrogation of both polyadenylation and termination.

Two models were proposed to explain these results. The first postulated that the emergence of polyadenylation sequences on the RNA triggers a change in the factors associated with the polymerase, which eventually results in termination. The second, also known as the 'torpedo' model, states that cleavage of the transcript is required to trigger termination. Recent evidence supports both models and, until the mystery is solved, only one thing is clear: it ain't over until pol II falls off.

Ekat Kritikou, Locum Associate Editor, Nature Reviews Molecular Cell Biology

\section{References and links}

ORIGINAL RESEARCH PAPERS Natori, S., Takeuchi, K. \& Mizuno, D. DNA-dependent RNA polymerase from Ehrlich ascites tumor cells. J. Biochem. 74, 1177-1182 (1973)|

Reines, D. Elongation factor-dependent transcript shortening by template-engaged RNA polymerase II. J. Biol. Chem. 267 3795-3800 (1992) | Izban, M. G. \& Luse, D. S. The RNA polymerase II ternary complex cleaves the nascent transcript in a $3^{\prime} \rightarrow 5^{\prime}$ direction in the presence of elongation factor SII. Genes Dev. 6, 1342-1356 (1992) | Wang, D. \& Hawley, D. K. Identification of a $3^{\prime} \rightarrow 5^{\prime}$ exonuclease activity associated with human RNA polymerase II. Proc. Natl Acad. Sci. USA 90 843-847 (1993) | Logan, J., Falck-Pedersen, E., Darnell, J. E. Jr \& Shenk, T. A poly(A) addition site and a downstream termination region are required for efficient cessation of transcription by RNA polymerase II in the mouse $\beta^{\text {maj-globin }}$ gene. Proc. Natl Acad. Sci. USA 84, 8306-8310 (1987)| Connelly, S. \& Manley, J. L. A functional mRNA polyadenylation signal is required for transcription termination by RNA polymerase II. Genes Dev. 2, 440-452 (1988)

FURTHER READING Birse, C. E., Minvielle-Sebastia, L.,

Lee, B. A., Keller, W. \& Proudfoot, N. J. Coupling termination of transcription to messenger RNA maturation in yeast. Science 280, 298-301 (1998)

\section{An alternative string theory}

The nucleosome hypothesis represents one of the great paradigm shifts in our understanding of eukaryotic gene expression. Formulated by Roger Kornberg in 1974, its key concept is that eukaryotic DNA is tightly packaged around a core of structural proteins - histones - to generate a nucleosome array that is fundamental for controlling gene expression.

Throughout the 1960s, it was largely accepted that chromatin was a linear strand of DNA coated with a simple repeated arrangement of five histones that packaged it into so-called ' 100 - $\AA$ fibres'. This view held that the DNA is encased within the histone protein, resulting in non-specific repression of transcription.

However, cracks in this view were beginning to surface. There was accumulating evidence to indicate that chromatin structures might not be so evenly distributed as originally thought. Evidence from X-ray diffraction, electron microscopy, and, in particular, a 1973 study by

\section{MILESTONE 10}

\section{Silent}

\section{remembrance}

Generally speaking, eukaryotic cells do not discard DNA as they differentiate. Cellular differentiation therefore has to be explained as the consequence of differential gene expression. So how are genes stably yet reversibly regulated? During the past 30 years, the direct modification of DNA by methylation has been shown to have a central role in repressing gene expression and transmitting the silenced state to daughter cells.

Among the founding papers in the field were the 1975 reviews by Arthur Riggs, and by Robin Holliday and John Pugh, who discussed the literature on DNA methylases in bacteria. Their models proposed that the properties of these enzymes - in particular, their preference for hemi-methylated substrates - made them ideally suited to establish stable differentiated states in the absence of genetic mutation. They further proposed that the sequence-specific binding of these enzymes would have a gene-regulatory role. This idea was not 
Hewish and Burgoyne in which chromatin was incubated with a nuclear deoxyribonuclease, led to the idea that histones might instead have a more periodic particulate structure. Another significant turning point was marked by the electron microscopy analyses of Olins and Olins, and Stanchfield and colleagues. They isolated a range of interphase nuclei and identified linear arrays of chromatin $\sim 70 \AA \AA$ in diameter. In some planes, the structures appeared connected by 15 - $\AA$ strands and were said to resemble 'beads on a string. At the time, however, these studies were met with considerable resistance - many suspected that the observed structures were merely artefacts of sample preparation.

It took the landmark analysis of Kornberg and Thomas to appreciate the significance of these observations, combine them with a detailed analysis of histone-histone interactions and arrive at the concept we now recognize as the nucleosome hypothesis. Using biochemistry, they found that the histone core consists of a tetramer of $\mathrm{H} 3$ and $\mathrm{H} 4$ subunits, and two copies each of $\mathrm{H} 2 \mathrm{~A}$ and $\mathrm{H} 2 \mathrm{~B}$. The next crucial advance was the finding that an $\mathrm{H} 3-\mathrm{H} 4$ tetramer, two $\mathrm{H} 2 \mathrm{~A}-\mathrm{H} 2 \mathrm{~B}$ oligomers and DNA were sufficient to recapitulate the known X-ray structure of

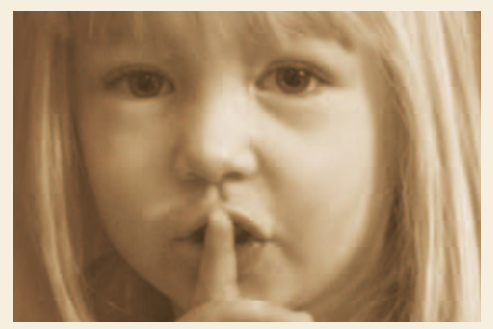

new, but its relevance to eukaryotic transcription had been in doubt, possibly owing to the apparent lack of DNA methylation in yeast, Caenorhabditis elegans and Drosophila melanogaster. Although not correct in all details, these influential syntheses focused attention on the potential role of methylation in gene expression.

By 1980, when a seminal paper by Peter Jones and Shirley Taylor was published, more substantial evidence of a role for DNA methylation in transcriptional repression had accumulated. Jones and Taylor introduced the use of cytidine analogues to prevent the methylation of cytosine residues in DNA, and directly linked changes in the patterns of methylation with changes in the differentiated state of the treated cells. The analogue 5-azacytidine was shown to be a potent inhibitor of DNA methylation, and is now used routinely to reactivate genes chromatin. Kornberg noted that chromatin contains one copy of each subunit per 100 base pairs of DNA. With the observed ratios of one tetramer and two oligomers from the biochemical analysis, he reasoned that the repeating unit of chromatin must therefore contain 200 base pairs of DNA. This hypothesis also fitted neatly with the Burgoyne study. Kornberg initially reported his findings at a Ciba Foundation Symposium in London, in April 1974. By the time he presented his hypothesis at a Gordon Research Conference in August of the same year, there were few who disagreed.

Subsequent studies by Finch et al. in 1977, and by Luger et al. and Davey et al. in 1997, reported the X-ray crystal structure of the nucleosome complex. These studies were no mean feat, as the large variation in post-translational modifications between species makes crystallization particularly difficult. What is remarkable, however, is that almost all the predictions of the original theory have been borne out by these structural studies. Indeed, to this day, the nucleosome hypothesis remains a cornerstone of modern biology.

Donald McDonald, Senior Production Editor, Nature Research Titles UK

that are silenced by methylation. It has also entered the clinic as a treatment for myelodysplastic syndrome.

Adrian Bird and colleagues subsequently provided further evidence linking DNA methylation and gene expression. In 1985, Bird and co-workers characterized the small fraction of the mouse genome that is frequently cleaved by a methylationsensitive restriction enzyme. These sequences, which would come to be known as $\mathrm{CpG}$ islands, are CpG-rich fragments with low or undetectable levels of methylation. The available literature indicated that $\mathrm{CpG}$ islands were typically located near the $5^{\prime}$ ends of genes, and the authors predicted correctly that genes might be associated with "methylationfree zones near sequences of regulatory significance."

Although all of these data pointed to a role for DNA methylation in regulating gene expression, the underlying molecular mechanisms were not discovered until the late 1990s. Perhaps the most farsighted prediction made by Riggs was that DNA methylation might affect gene expression indirectly by changing the affinity of sequence-specific DNA-binding proteins for their target sites. Definitive evidence came from the laboratories of Bird and Alan Wolffe in 1998. Each group showed that Mecp2, which had been shown to bind to methylated DNA and repress transcription, does so by recruiting a histone deacetylase complex that alters chromatin structure. Although the exact nature of this crosstalk between DNA and histone epigenetic marks (see Milestone 22) is still being worked out, the importance of DNA methylation as a stable regulator of transcriptional repression has been firmly established.

Alan Packer, Senior Editor, Nature Genetics

References and links

ORIGINAL RESEARCH PAPERS Riggs, A. D.

$X$ inactivation, differentiation, and DNA methylation. Cytogenet. Cell Genet. 14, 9-25 (1975) | Holliday, R. \& Pugh, J. E. DNA modification mechanisms and gene activity during development. Science 187, 226-232 (1975) | Jones, P. A. \& Taylor, S. M. Cellular differentiation, cytidine analogs, and DNA methylation. Cell 20, 85-93 (1980) | Bird, A., Taggart, M., Frommer, M., Miller, O. J. 8 Macleod, D. A fraction of the mouse genome that is derived from islands of nonmethylated, $\mathrm{CpG}$-rich DNA. Cell 40, 91-99 (1985) | Nan, X. et al. Transcriptional repression by the methyl-CpG-binding protein MeCP2 involves a histone deacetylase complex. Nature $\mathbf{3 9 3}$, 386-389 (1998) | Jones, P. L. et al. Methylated DNA and MeCP2 recruit histone deacetylase to repress transcription. Nature Genet. 19, 187-191 (1998) FURTHER READING Wolf, S. F. \& Migeon, B. R. Clusters of CpG dinucleotides implicated by nuclease hypersensitivity as control elements of housekeeping genes. Nature 314, 467-469 (1985) | Li, E., Bestor, T. H. \& Jaenisch, R. Targeted mutation of the DNA methyltransferase gene results in embryonic lethality. Cell 69, 915-926 (1992) | Jones, P. A. \& Laird, P. W. Cancer epigenetics comes of age. Nature Genet. 21, 163-167 (1999) 